\title{
Über die Absorptionsspektren elektrostatischer Komplexionen dreiwertiger Übergangselemente mit oktaedrischer Symmetrie
}

\author{
Farbe und Konstitution von Komplexverbindungen \\ 5. Mitteilung \\ Von Hermann Hartmann und Hans Ludwig Schläfer \\ Aus dem Institut für physikalische Chemie und dem Institut für anorganische Chemie \\ der Universität Frankfurt a. M. \\ (Z. Naturforschg. 6a, 760-763 [1951]; eingegangen am 4. September 1951)
}

\begin{abstract}
Es wird gezeigt, daß man die langwelligen Absorptionsspektren der magnetisch normalen oktaedrischen Komplexionen mit den Übergangsmetallionen $\mathrm{Ti}^{3+}, \mathrm{V}^{3+}, \mathrm{Cr}^{3+}, \mathrm{Mn}^{3+}$ und $\mathrm{Fe}^{3+}$ verstehen kann, wenn man annimmt, daß die schwachen langwelligen Banden durch Übergänge zwischen den Aufspaltungsprodukten der jeweiligen Grundterme entstehen, wobei die Aufspaltungen durch das elektrostatische Feld der Liganden zustande kommen.
\end{abstract}

Es: s ist gezeigt worden, daß bei dem zweifellos elektrostatisch aufzufassenden $\operatorname{Ion}\left[\mathrm{Ti}\left(\mathrm{H}_{2} \mathrm{O}\right)_{6}\right]^{3+}$ das von den Liganden herrührende elektrische Feld den ${ }^{2} \mathrm{D}$-Grundterm des freien $\mathrm{Ti}^{3+}$-Ions in zwei Terme aufspaltet, von denen jeder zu einer bestimmten irreduziblen Darstellung der Symmetriegruppe des Ions $\left(\mathrm{O}_{\mathrm{h}}\right)$ gehört $^{1}$. Eine Abschätzung der Größe der Aufspaltung mit Hilfe einer Störungsrechnung hat ergeben, daß ein Übergang zwischen den beiden Aufspaltungsprodukten einer Absorptionsfrequenz im sichtbaren Spektralgebiet entsprechen müßte. Da im einfachen Fall des $\mathrm{Ti}^{3+}$ sonst keine tiefliegenden Terme existieren, konnten wir die im sichtbaren Spektralbereich liegende schwache Absorptionsbande diesem Übergang mit hoher Wahrscheinlichkeit zuordnen und damit eine Erklärung der sonst rätselhaften Farbabsorption der wäßrigen Lösungen von Titan(III)-Salzen geben ${ }^{2}$.

Wir wollen in dieser Mitteilung von dem bei der Untersuchung der Titan(III)-Spektren gewonnenen Standpunkt aus den langwelligen Teil der Absorptionsspektren der oktaedrischen elektrostatischen Komplexionen mit den Zentralionen: $\mathrm{V}^{3+}, \mathrm{Cr}^{3+}$, $\mathrm{Mn}^{3+}$ sowie $\mathrm{Fe}^{3+}$ diskutieren. Dazu ist es nötig, daß wir zunächst die möglichen Termaufspaltungen qualitativ betrachten.

Die Symbole S, P, D usw. können in bekannter Weise als Symbole für die irreduziblen Darstellungen

1 1. Mitt.: F. E. Il s e u. H. H a r tmann, Z. physik. Chem. 197, 239 [1951].

2 2. Mitt.: H. Hartmann u. H. L. S chläf e r, Z. physik. Chem. 197, 116 ff. [1951]. der vollen Kugeldrehgruppe aufgefaßt werden. Wenn man eine dieser Darstellungen betrachtet und aus ihr diejenigen Matrices herausgreift, die Symmetrieoperationen entsprechen, die auch in der Oktaedergruppe $\mathrm{O}$ vorkommen, so bilden diese Matrices eine im allgemeinen noch reduzible Darstellung der Oktaedergruppe O. Durch Bestimmung der irreduziblen Darstellungen der Gruppe $\mathrm{O}$, die in der gebildeten reduziblen Darstellung enthalten sind, erhält man Auskunft darüber, zu welcher Darstellung von $\mathrm{O}$ die Terme gehören, die aus einem Term des kugelsymmetrischen Gebildes entstehen, der zu der betrachteten irreduziblen Darstellung der Kugeldrehgruppe gehört und wieviele Terme es sind. Dasselbe Verfahren kann man anwenden, wenn man nicht von den Darstellungen der Kugeldrehgruppe, sondern von denen der Kugeldrehspiegelungsgruppe ausgeht, wenn man also untersuchen will, welche Terme bei Einwirkung eines Feldes von der Symmetrie $\mathrm{O}_{\mathrm{h}}$ aus einem Term des kugelsymmetrischen Falles hervorgehen, von dem man zusätzlich noch weiß, ob es ein gerader oder ungerader Term ist. Da wir es im folgenden nur mit geraden Termen zu tun haben, die wir durch den Index g charakterisieren, erhalten wir in der angegebenen Weise die in Tab. 1 angegebenen Aufspaltungsbilder.

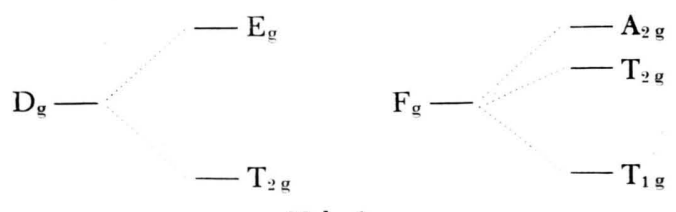

Tab. 1. 
[Die Reihenfolge der Terme läßt sich gruppentheoretisch nicht ermitteln. In der Darstellung sind die Reihenfolgen angegeben, die eine Störungsrechnung bei $\mathrm{Ti}^{3+}\left({ }^{2} \mathrm{D}\right)$ und $\mathrm{V}^{3+}\left({ }^{3} \mathrm{~F}\right)$ ergibt. Die Termordnung bei $\mathrm{Cr}^{3+}\left({ }^{4} \mathrm{~F}\right)$ z. B. ist gerade umgekehrt wie die bei $\mathrm{V}^{3+}$.]

Die tiefliegenden Terme der freien Ionen $\mathrm{Ti}^{3+}$ bis $\mathrm{Fe}^{3+}$ gehören jeweils zur Elektronenkonfiguration $\mathrm{d}^{\mathrm{n}}$ mit $\mathrm{n}=1$ bis 5 . Sie sind also alle gerade. Wir haben nun von vorneherein zwei Aufspaltungsfälle bei der Einwirkung eines Feldes der Symmetrie $\mathrm{O}_{\mathrm{h}}$ zu unterscheiden:

1. Der Grundterm des Komplexions geht aus dem Grundterm des freien Ions hervor, er ist mit anderen Worten ein Aufspaltungsprodukt dieses Terms.

2. Ein Aufspaltungsprodukt eines im freien Ion höher liegenden Terms kommt bei der Einschaltung des Störungsfeldes der Liganden unter die Aufspaltungsprodukte aller anderen Terme zu liegen. Wenn der Term des freien Ions, aus dem der Grundterm des Komplexions in dieser Weise hervorgegangen ist, andere Multiplizität besitzt als der Grundterm des freien Ions, muß das magnetische Verhalten des Komplexions „anomal“ sein.

Welcher der beiden Fälle jeweils vorliegt, läßt sich nur durch eine an die gruppentheoretischen Überlegungen sich anschließende Störungsrechnung entscheiden. Immerhin gibt aber „normales“ magnetisches Verhalten einen, wenn auch nicht völlig eindeutigen, so doch sehr starken Hinweis darauf, daß Fall 1 vorliegt. Die von uns hier in Betracht gezogenen Komplexionen der Ionen $\mathrm{Ti}^{3+}$ bis $\mathrm{Fe}^{3+}$ zeigen „normales" magnetisches Verhalten.

Wenn Fall 1 vorliegt, interessieren für das optische Verhalten wegen der Auswahlregel, daß praktisch nur Übergänge zwischen Termen gleicher Multiplizität vor sich gehen, nur Terme, die dieselbe Multiplizität besitzen wie der Grundterm des Komplexions. Diese kann man sich in Gruppen zusammengefaßt denken, von denen jede beim Einschalten des Störfeldes aus einem Term des freien Ions entstanden ist. Terme, die $\mathrm{zu}$ verschiedenen Gruppen, aber zur gleichen irreduziblen Darstellung gehören, können sich nicht überkreuzen, beeinflussen aber ihre Lage gegenseitig in Gestalt einer Konfigurationswechselwirkung. Das rührt daher, daß unabhängig von der Gruppenzugehörigkeit alle Terme zu einer bestimmten irreduziblen Darstellung aus einem Säkularproblem der Störungsrechnung hervorgehen. Wenn im freien Ion die angeregten Terme, die dieselbe Multiplizität haben wie der Grundterm, von diesem weit genug entfernt sind, dürften Überschneidungen von Termen, die $\mathrm{zu}$ verschiedenen Gruppen und verschiedenen irreduziblen Darstellungen gehören, kaum vorkommen. Wir haben deshalb, obwohl wir bisher Störungsrechnungen nur für $\mathrm{Ti}^{3+}, \mathrm{V}^{3+}$ und $\mathrm{Cr}^{3+}$ durchgeführt $^{1,3}$ und die ausgesprochene Vermutung insbesondere beim $\mathrm{V}^{3+}$ bestätigt gefunden haben, bei der folgenden Diskussion angenommen, daß keine Überschneidungen stattfinden. Unter dieser Voraussetzung ist die Zahl der langwelligen Banden der Komplexionen, wie aus der Tab. 1 hervorgeht, einfach durch die Bahndrehimpulsquantenzahl bzw. die diesem Term zugehörige irreduzible Darstellung der Kugeldrehgruppe bestimmt. Die langwelligen Banden entstehen durch Übergang von dem tiefsten zu den anderen Aufspaltungsprodukten des Grundterms des freien Ions. Sie sind schwach, weil alle diese Übergänge, wie man leicht nachrechnen kann, als reine Elektronenübergänge verboten sind und nur in Kombination mit Schwingungsübergängen vonstatten gehen können.

Elektrostatische Komplexionen oktaedrischer Symmetrie der Ionen $\mathrm{Ti}^{3+}$ bis $\mathrm{Mn}^{3+}$ sollten also eine schwache langwellige Bande aufweisen, wenn der Grundterm des freien Ions ein D-Term ist, und sie sollten zwei schwache langwellige Banden aufweisen, wenn der Grundterm des freien Ions ein F-Term ist. Die Grundterme der freien Ionen sind in Tab. 2 angegeben.

$\begin{array}{ll}\mathrm{Ti}^{3+} & { }^{2} \mathrm{D} \\ \mathrm{V}^{3+} & { }^{3} \mathrm{~F} \\ \mathrm{Cr}^{3+} & { }^{4} \mathrm{~F} \\ \mathrm{Mn}^{3+} & { }^{5} \mathrm{D} \\ \mathrm{Fe}^{3+} & { }^{6} \mathrm{~S}\end{array}$

Tab. 2. Grundterme der freien Ionen.

Wie wir in der zweiten Mitteilung ${ }^{2}$ gezeigt haben, ist bei dem Ion $\left[\mathrm{Ti}\left(\mathrm{H}_{2} \mathrm{O}\right)_{6}\right]^{3+}$ die Theorie in Übereinstimmung mit dem Experiment, da dieses Ion eine schwache langwellige Bande besitzt. $\mathrm{Da} \beta$ das Ion $\left[\mathrm{V}\left(\mathrm{H}_{2} \mathrm{O}\right)_{6}\right]^{3+}$ zwei schwache langwellige Banden aufweist, haben $\mathrm{Furman}$ und $\mathrm{Gar}$ $n$ e $r^{4}$ gezeigt. Ebenso fanden wir bei der Untersuchung der kristallisierten .Vanadin(III)-alaune $\left[\mathrm{NH}_{4} \mathrm{~V}\left(\mathrm{SO}_{4}\right)_{2} \cdot 12 \mathrm{H}_{2} \mathrm{O}\right.$ und $\left.\mathrm{CsV}\left(\mathrm{SO}_{4}\right)_{2} \cdot 12 \mathrm{H}_{2} \mathrm{O}\right]$ zwei schwache Banden ${ }^{5}$. Wir konnten außerdem in der

3 3. Mitt.: F. E. Il s e u. H. H a rtmann, Z. Naturforschg. 6 a, 751 [1951].

4 S. C. F u rma n u. C. S. G a r n e r, J. Amer. chem. Soc. 72, 1785 [1950].

5 4. Mitt.: H. Hartmann u. H. L. S chläf er, Z. Naturforschg. 6a, 754 [1951]. 
4. Mitteilung ${ }^{5}$ zeigen, daß die ebenfalls oktaedrischen Ionen $\left[\mathrm{V}(\mathrm{alk})_{6}\right]^{3+}($ alk. $=$ Methanol, Äthanol und isoButanol) auch zwei schwache langwellige Banden besitzen und daß die geringfügige Verschiebung der beiden Maxima gegenüber der Lage beim Hexaquovanadin(III)-Ion qualitativ im Sinne der Störungsrechnung erklärt werden kann. Die elektrostatischen Komplexionen oktaedrischer Symmetrie von $\mathrm{Cr}^{3+}$ sollten zwei schwache Banden zeigen. Nach Messungen von $\mathrm{Tsuchida}$ und $\mathrm{Kobayashi}{ }^{6}$ an $\left[\mathrm{Cr}\left(\mathrm{H}_{2} \mathrm{O}\right)_{6}\right] \mathrm{Cl}_{3}$ ist dies auch der Fall. Besonders interessant war für uns der Fall des $\mathrm{Mn}^{3+}$, das einen ${ }^{5} \mathrm{D}-\mathrm{Term}$ als Grundterm besitzt, und bei dessen oktaedrischen Komplexen deshalb wieder nur eine schwache Bande zu erwarten war. Da in Mangan(III)Salzlösungen wegen der Bildung von Acidoverbindungen sehr undurchsichtige Verhältnisse herrschen, blieb als einziges geeignetes Objekt für eine spektroskopische Untersuchung der Caesium-mangan(III)alaun. Über die Herstellung dieser äußerst instabilen Substanz und die Durchführung und genauen Ergebnisse der optischen Untersuchung werden wir an anderer Stelle berichten. Wir kennen aber jetzt schon sicher den sichtbaren Teil des Absorptionsspektrums, in dem eine schwache Bande liegt, und wir können außerdem mit Sicherheit angeben, daß vor dem starken Absorptionsanstieg im kurzwelligen UV keine schwache Bande mehr auftritt.

In der jüngsten Zeit haben außerdem $S \mathrm{ch}$ e f f e r und $\mathrm{Hammaker}{ }^{7}$ das Absorptionsspektrum des Ions $\left[\mathrm{MnF}_{6}\right]^{3-}$ ermittelt und dabei eine schwache langwellige Bande gefunden, deren Maximum bei $460 \mathrm{~m} \mu$ liegt, während das des Caesiummangan(III)alauns von uns bei $475 \mathrm{~m} \mu$ gefunden wurde. Auch beim $\mathrm{Mn}^{3+}$ sind also die Beobachtungen in Übereinstimmung mit unseren theoretischen Vorstellungen.

Der Fall des Eisen(III)-Ions, das durch Anlagerung von $6 \mathrm{H}_{2} \mathrm{O}$-Molekülen ein magnetisch normales Komplexion ergibt, ist besonders interessant, weil der aus der Konfiguration $\mathrm{d}^{5}$ hervorgehende Grundterm des freien $\mathrm{Fe}^{3+}$-Ions ein ${ }^{6} \mathrm{~S}$ ist und aus derselben Konfiguration sonst nur Terme niedrigerer Multiplizität hervorgehen. Ein S-Term kann natürlich beim Hinzukommen des Komplexfeldes nicht aufspalten, so daß für den langwelligen Teil des Absorptionsspektrums des Ions $\left[\mathrm{Fe}\left(\mathrm{H}_{2} \mathrm{O}\right)_{6}\right]^{3+}$ nur Interkombinationsbanden zwischen Termen verschiedener Multi-

6 R. Tsuchida u. M. Kobay a shi, Bull. chem. Soc. Japan 13, 476 [1938].

7 E. R. S c h effer u. E. M. H a m m a k er, J. Amer. chem. Scc. 72, 2575 [1950]. plizität in Frage kommen. Diese müßten aber wesentlich schwächer sein als die schwachen Banden der übrigen hier betrachteten Komplexe. Tatsächlich zeigt das Absorptionsspektrum dieses Ions nach Messungen von v. Kiss und Mitarbeitern ${ }^{8}$ sowie Rabinowitch

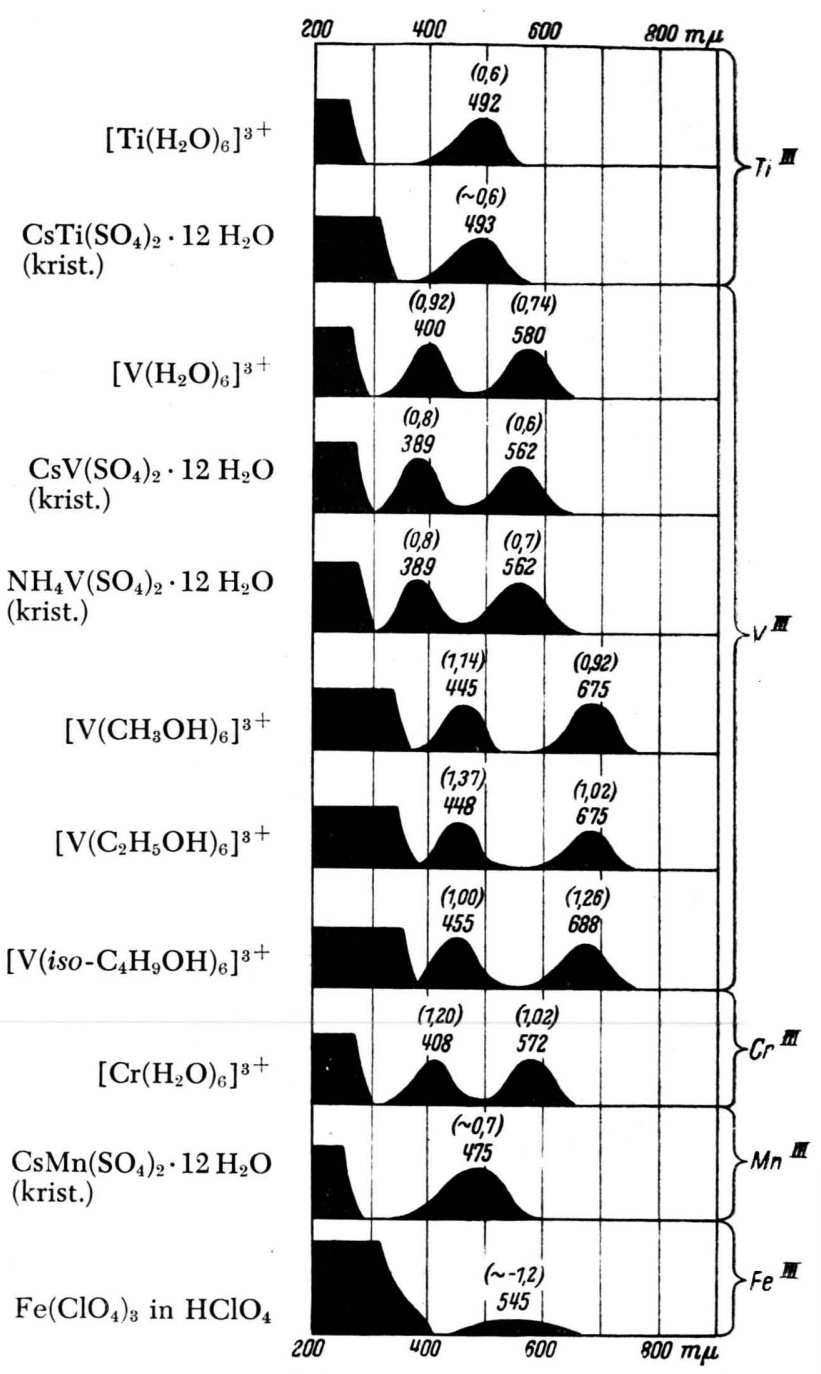

Abb. 1. Schematische Darstellung der Absorptionsspektren von Komplexionen mit $\mathrm{O}_{\mathrm{h}}$-Symmetrie. (Zahlen in Klammern bedeuten $\log \varepsilon$ für das Bandenmaximum.)

und Stockmayer ${ }^{9}$, das wir zusammen mit den Absorptionsspektren der schon besprochenen Ionen in Abb. 1 schematisch wiedergegeben haben, eines zwischen 350 und $400 \mathrm{~m} \mu$ beginnenden starken UY -

8 A. v. Kis s, J. A braham u. I. Heged üs, Z. anorg. allg. Chem. 244, 99 [1940].

9 E. Kabinowitch u. W. H. Stockmayer J. Amer. chem. Soc. 64, 335 [1942]. 
Anstieg; im langwelligen Gebiet aber nur eine $\mathrm{Ab}$ sorption deren $\log \varepsilon$ den Wert - 1,2 nie überschreitet, während die $\log \varepsilon$-Werte der schwachen langwelligen Banden der übrigen hier besprochenen Komplexionen alle größenordnungsmäßig bei +1 liegen. Die langwelligen Absorptionsbanden des $\left[\mathrm{Fe}\left(\mathrm{H}_{2} \mathrm{O}\right)_{6}\right]^{3+}$ sind also in Úbereinstimmung mit der Theorie um mindestens den Faktor 100 schwächer als die der übrigen vergleichbaren Ionen.

Beim hydratisierten $\mathrm{Mn}_{\text {aq. }}^{2+}$-Ion liegen, da dieses Ion ebenfalls die Konfiguration $\mathrm{d}^{5}$ besitzt, ganz analoge Verhältnisse vor, und tatsächlich zeigt das Absorptionsspektrum dieses Ions, worauf wir aber an dieser Stelle noch nicht näher eingehen wollen, im langwelligen Teil nur Banden, deren Intensität etwa gleich der der langwelligen Bande des hydratisierten $\mathrm{Fe}_{\text {aq. }}^{3+}$-Ions ist. $\mathrm{Fe}^{3+}$ und $\mathrm{Mn}^{2+}$ spielen bei den Ionen der Úbergangsmetalle hinsichtlich des optischen Verhaltens also dieselbe Rolle wie das $\mathrm{Gd}^{3+}$ in der Reihe der Seltenen Erden.

Die Deutung, die De uts chbe in ${ }^{10}$ für die Entstehung der beiden Chrombanden gegeben hat, hal-

10 O. De u t s c h b e in, Z. Physik 77, 489 [1932], und andere Arbeiten. ten wir für unzutreffend, da die Aufspaltungen der Terme der freien Ionen, die Deutschbein überhaupt nicht in Betracht zieht, nach den Ergebnissen unserer Störungsrechnungen so groß sind, daß Übergänge zwischen den Aufspaltungsprodukten schon zu Absorptionen im sichtbaren Spektralgebiet Veranlassung geben. Außerdem läßt sich, abgesehen davon, daß Deutschbein den tiefliegenden ${ }^{4} \mathrm{P}-\mathrm{Term}$ des $\mathrm{Cr}^{3+}$. Ions nicht berücksichtigt, leicht zeigen, daß seine Deutung bei der Übertragung seiner Grundvorstellung auf die übrigen Komplexionen der Übergangsmetalle, die wir hier diskutiert haben, zu Ergebnissen führt, die nicht in Übereinstimmung mit den Beobachtungen sind.

Unsere bisherigen Ergebnisse haben uns ermutigt, die theoretische Untersuchung der in den schönen Arbeiten von $\mathrm{L}$ in hard ${ }^{\mathbf{1 1}}$ mitgeteilten Bandenaufspaltungen bei Komplexionen niedrigerer Symmetrie von unserem Standpunkt aus in Angriff zu nehmen. Wir werden darüber in den folgenden Abhandlungen dieser Reihe berichten.

11 M. Linhard u. M. W e ig e l, Über Komplexverbindungen VIII, Z. anorg. allg. Chemie (im Druck) sowie vorhergehende Arbeiten.

\title{
Methylcellulosen verschiedenen Polymerisationsgrades in ihrer Auswirkung auf polarographische Reduktionen
}

\author{
Von Ludwig Holleck und Herbert J. Exner \\ Aus dem Institut für Physikalische Chemie der Universität Freiburg i. Br. \\ (Z. Naturforschg. 6 a, 763-767 [1951]; eingegangen am 17. September 1951)
}

Bei der polarographischen Reduktion 0,001-mol. gepufferter $p$-Nitranilin-Lösungen bei $p_{\mathrm{H}} 11,0$ ergab sich bei Zusätzen von Methylcellulose eine Abhängigkeit der Beeinflussung der Stromspannungskurven vom Polymerisationsgrad des Zusatzes. Es kamen Präparate vom Polymerisationsgrad 125, 200, 315, 500, 590 zur Anwendung.

Bei gleicher Gewichtskonzentration zeigte das stärker abgebaute Produkt eine größere Wirkung als das höhermolekulare. Aus diesem Zusammenhang folgt, daß die Fadenmoleküle der Methylcellulose mit ihrer Molekülachse senkrecht zur Oberfläche des Quecksilbertropfens adsorbiert werden.

Für die Verwendung zur Maximumdämpfung ergibt sich danach, daß Methylcellulosen höheren Polymerisationsgrades bis zu größeren Gewichtskonzentrationen anwendbar sind, ohne $\mathrm{da} ß$ es zu einer Veränderung der Kurvengestalt kommt.

$\mathrm{E}$ ine häufige Erscheinung bei der Aufnahme polarographischer Stromspannungskurven sowohl anorganischer als auch organischer Verbindungen ist das Auftreten sog. Maxima. Man versteht darunter das Ansteigen der Stromstärke, z. Tl. weit über den Diffusionsstrom hinaus, die dann bei einem bestimmten Potential fast momentan auf den Betrag des Dif- fusionsstromes zurückgeht. Um nun für die analytische Auswertung zu einer einwandfreien Ausmessung der Reduktionsstufen zu kommen, sucht man die auftretenden Maxima zu unterdrücken. Dies geschieht in der polarographischen Praxis meist durch oberflächenaktive Stoffe, die an der Quecksilberoberfläche der Tropfkathode adsorbiert werden. Als solche Maxima- 